\section{Responsibility of genetic testing}

To the Editor:

The recent publications containing data on tens of thousands of genetic tests performed on individuals of Ashkenazi Jewish descent reflect the arrival of a new era of genetic testing and awareness. This era has presented physicians with new medical and moral challenges and responsibilities. With these challenges before us, I would ask that the medical community consider whether physicians are adequately trained to interpret and then explain to their patients the importance and significance of the genetic testing being performed. I would further suggest that guidelines be developed that address the social and psychological impact these results will have on the patient.

At Dor Yeshorim, we have screened nearly 200,000 blood samples for as many as 10 genetic diseases prevalent in the Ashkenazi Jewish community; this has been done in a manner that ensures confidentiality and avoids any social or psychological stigma that may be associated with knowing that one is a carrier for a genetic disease. Our sensitivity to our clientele is in sharp contrast to what many expectant mothers in our community report experiencing from their obstetricians. I understand what motivates the physicians and I support their diligence, but this diligence must be accompanied by knowledge, expertise, understanding, and compassion. Patients must be provided with information that is accurate, valuable, and useful. To test and then inform a pregnant woman that she is a carrier for a devastating genetic disease is, in my view, cruel. Her carrier status is irrelevant if her spouse is not a carrier for the same disease. Among those of Ashkenazi Jewish descent, where one in approximately five individuals is a carrier for a serious genetic disease, far too many expectant mothers are informed of their carrier status only to learn, several agonizing weeks later, that their husband is not a carrier and that their unborn child is not at risk. For a population with a high carrier frequency, if testing is performed, it should be performed on both parents so the results provided to the expectant mother are conclusive.

As not all genetically transmitted mutations present a serious risk to the child, physicians must carefully consider which diseases they will test for. Testing and then informing parents that they are carriers for Gaucher disease is of little value. As homozygosity for Gaucher disease would not justify termination of a pregnancy, this data simply serves to alarm, confuse, and upset those tested.

Physicians must keep in mind that not all genetic tests are of equal reliability. Whereas DNA-based genetic testing in most laboratories is extremely accurate and reliable, results generated using enzyme-based assays can be more problematic. For example, the enzyme-based assay for Tay Sachs requires that the blood drawn be handled under controlled conditions. Placing a blood sample for enzyme-based Tay Sachs testing in a metal box on a warm day is almost certain to yield faulty or, at the very least, inconclusive results. Transmitting to an expectant mother her possible carrier status based on such a test is certain to generate fear and concern-emotional states that are certainly not beneficial to the health and well-being of either mother or fetus. I understand the rationale for using the enzyme-based test for Tay Sachs in the general population, as genetic testing for some of the mutations is not readily available. I question the necessity of ordering enzyme-based tests for individuals of Ashkenazi Jewish descent when the DNAbased tests are readily available. I suspect that this test is often ordered as a result of a lack of understanding on the part of some physicians.

Testing a patient for a genetic disease comes with significant responsibility. I encourage physicians prescribing such testing to do it with the patient's informed consent and that they transmit the information to their patient in an informative, accurate, and compassionate manner. This will ensure that the patient understands the disease for which they are a carrier, they have a clear understanding of what it means to be a carrier for a genetic disorder, and they know what their medical options are should both parents carry the same genetic disorder. Finally, I ask that all who do genetic testing and genetic counseling be sensitive to the fact that, for some couples, the medical options that they will consider, upon learning that they are carriers for the same disorder or that a fetus is potentially homozygous for a devastating genetic disorder, are limited.

Rabbi Joseph Ekstein
Executive Director
Dor Yeshorim
Committee for Prevention of Jewish Genetic Diseases
Brooklyn, NY

Rabbi Joseph Ekstein Dor Yeshorim Brooklyn, NY 